\title{
Historical Evolution of the
} Anticoagulant-Antiplatelet Therapy in Patients with Mitral Valve Disease Associated to Atrial Fibrillation or Mechanical Valve Prosthesis. Role of Omeprazole

\author{
Francisco Perez Gómez, Ramon Bover \\ University Hospital Clínico San Carlos, Madrid, Spain \\ Email:fpgomez@salud.madrid.org,ramonbover@yahoo.es
}

How to cite this paper: Gómez, F.P. and Bover, R. (2020) Historical Evolution of the Anticoagulant-Antiplatelet Therapy in Patients with Mitral Valve Disease Associated to Atrial Fibrillation or Mechanical Valve Prosthesis. Role of Omeprazole. World Journal of Cardiovascular Diseases, 10, 698-706.

https://doi.org/10.4236/wjcd.2020.1010067

Received: August 19, 2020

Accepted: October 19, 2020

Published: October 22, 2020

Copyright $\odot 2020$ by author(s) and Scientific Research Publishing Inc. This work is licensed under the Creative Commons Attribution International License (CC BY 4.0).

http://creativecommons.org/licenses/by/4.0/

\begin{abstract}
In this review article we analyze the historical evolution of anticoagulant and antiplatelet therapies in the treatment of atrial fibrillation plus complicated heart diseases. Its combined use, compared with anticoagulation alone, has been widely used to significantly reduce thromboembolic risk. Major bleeding risk has been usually registered during combined antithrombotic therapy, which is mainly due to gastric bleeding, but enteric coating antiplatelet tablets can effectively protect against gastric mucosal damage, and the addition of proton-pump inhibitors (omeprazole) can avoid it.
\end{abstract}

\section{Keywords}

Ischemic Heart Disease, Anticoagulant-Antiplatelet Therapy

\section{Introduction}

Thrombus formation in atrial fibrillation is the result of two simultaneous and necessary processes: 1) blood coagulation and 2) platelet activity. The role of both processes, which we describe later, is different according to the intensity of blood flow velocity and turbulence: a) in situations of high blood flow, as may happen in mitral stenosis and arterial cerebral or myocardial stenosis, the role of platelet activity is predominant, and b) in situations of low blood flow (atrial fibrillation) the main factor for left atrium thrombus is coagulation, although the association of mitral valve disease or mechanical valve prosthesis highly increas- 
es the role of platelet aggregation due to increased turbulent flow. We dedicate this article to the prevention of thrombus in atrial fibrillation associated to ischemic heart disease, to mitral stenosis or mechanical prosthetic heart valves.

\section{Physiopathology of Thrombus Formation}

Thrombus formation in atrial fibrillation (AF) is the result of two simultaneous processes: 1) coagulation, which finishes with the formation of an active thrombin responsible to transform fibrinogen to a stable fibrin net; 2) platelet activity following two ways, one which generates thromboxane and later prostacyclin to effectively collaborate to thrombin activation, and the other to generate ADP and later glycoproteins to actively contribute to form platelet aggregates.

1) Coagulation. Macfarlane [1] presents in 1994 the, so called, classic coagulation cascade: thrombin, formed from prothrombin thanks to the collaboration of the complex factor VII-tissular factor and factor Xmay finished the coagulation cascade and transform some fibrinogen into fibrin net, but this is unable to form an stable thrombus.

Schafer [2] and Monroe [3], modified the classic cascade and present the new coagulation cascade, which finishes with the formation of an activated thrombin capable to transform fibrinogen into an efficient fibrin net. Thrombin and platelets mutually activate themselves with the collaboration of several factors of the medium phase of the coagulation to activate great amount of thrombin capable to transform great amount of fibrinogen into an active fibrin net.

2) Activated platelets, apart from their mentioned role to activate thrombin, are also responsible for the generation of glycoproteins, which are responsible for the formation of platelet aggregates. While isolated platelets can pass through the small holes of the fibrin net, the aggregates of many platelets cannot pass and contribute to a stable thrombus.

In summary, activated platelet necessarily participates in both mentioned processes for thrombus formation: 1) the activation of enough amount of thrombin, able to form with the fibrinogen a stable fibrin net and 2) the generation of glycoproteins and platelet aggregates. These two processes justify the important role of platelets in thrombus formation.

\section{Combined Anticoagulant-Antiplatelet Therapy}

Most of the trials to prevent thrombus formation used warfarin as vitamin $\mathrm{K}$ antagonist controlled for an INR ratio between 2 and 4.5 according to the thrombotic risk. More recently thrombin inhibitors were incorporated. To inhibit platelet activation aspirin and occasionally triflusal were used to inhibit the thromboxane line. Clopidogrel alone or combined with aspirin has also being used.

\section{First Trials}

Chesebro and Fuster [4] were the first investigators to combine anticoagulants 
(warfarin at therapeutic level) and antiplatelets (aspirin at a dose of $500-1000$ $\mathrm{mg} / 24 \mathrm{~h}$.) in patients with mechanical valve prosthesis. The trial was prematurely stopped due to the high incidence of major bleeding events.

Turpie et al. [5], using a combination of aspirin $100 \mathrm{mg} /$ day + complete anticoagulation (INR 2-3) versus anticoagulation alone, showed that combined therapy significantly reduced the risk of embolism in patients with heart valve prosthesis without increasing the risk of major bleeding, although minor bleeding was increased.

Two similar trials, one realized in USA [6] and the other in Copenhagen [7] combined a dose of aspirin ( $300-350 \mathrm{mg} / \mathrm{d})$ and warfarin for a very low INR level (mean value 1.3) in patients with AF. Both failed to prevent thromboembolism.

In those years, some other trials compared low dose aspirin plus low intensity anticoagulation versus warfarin or aspirin alone in patients with ischemic heart disease. Using low dose of aspirin, the major bleeding risk was not increased, but there was some tendency to gastrointestinal bleeding and endoscopy did not show gastric ulcer.

After these initial trials, the first conclusion was that the level of anticoagulation in combined therapy should be therapeutic (INR 2-3) or higher and the dose of aspirin should be low, but the level was not yet established.

\section{Trials at the End of $20^{\text {th }}$ Century}

Turpie et al. [5] used warfarin for an INR 3-4.5 were compared with the same level of anticoagulation plus $100 \mathrm{mg}$ of enteric coating aspirin in patients with heart valve prosthesis. Combined therapy significantly reduced the risk of embolism and total mortality. There was a non-significant increase of major and minor bleeding risk.

Meschengieser et al. [8], compare doral anticoagulation (INR 2.5-3.5) plus low dose aspirin $(100 \mathrm{mg} /$ day) versus high intensity oral anticoagulation alone (INR 3.5-4.5) in patients with mechanical prosthetic heart valves. There were less thromboembolic events in the combined group, but increased gastrointestinal bleeding was registered as it was shown in other trials.

\section{Trials at the Beginning of the $21^{\text {st }}$ Century}

Massel et al. [9] published in 2001 a Meta-Analysis about the risks and benefits of adding anti-platelet therapy to warfarin versus warfarin or aspirin alone among patients with prosthetic heart valves. The dose of aspirin was progressively reduced from $1000-500 \mathrm{mg} /$ day to less than 300 and the level of anticoagulation also tended to be reduced to an INR of 2-3. After these changes the thromboembolicrisk and total mortality was diminished during combined therapy, but the incidence of major bleeding was slightly higher than with anticoagulants alone. When the dose of aspirin was reduced to $100 \mathrm{mg} /$ day in combination with moderate level of anticoagulant therapy the mayor bleeding risk was 
similar to that registered in the high dose anticoagulant alone group [8].

Lechat et al. [10] used aspirin 100 plus warfarin (INR 2-2.5) vs. anticoagulant therapy alone (INR 2-3). Aspirin was not enteric coating. Total bleeding events were 13 ( 9 of them non-severe). One of the 3 major bleedings was registered in the anticoagulant alone group and 3 majors were registered in the combined group (1 gastric, 1 retroperitoneal and 1 post-traumatic). 8 non-severe bleedings were registered in the combined group and one in the anticoagulant alone. The trial was stopped due to the incidence of bleeding complications. There was no information about the location of the minor bleeding events.

Dentali et al. [11] analyzed by meta-analysis of randomized trials the benefit of combined versus anticoagulant alone therapy in patients with cardiovascular diseases. The thromboembolic risk was lower in patients receiving combined therapy, mainly in those with cardiac valve disease. The benefit was only significant in patients with cardiac valve prosthesis. The incidence of fatal thromboembolism was also lower in this group of patients. Considering all groups of patients, the incidence of major bleeding was non-significantly lower in the group receiving anticoagulant therapy alone and the incidence of intracranial or fatal bleeding was similar in both groups.

It is generally accepted by experts in gastroenterology [12] [13] [14] [15] the relationship between antiplatelet drugs and major gastric bleeding caused by gastric ulcer and its protection with omeprazole. It was also shown that superficial mucosal erosions cause only microscopic bleeding detected either in gastric or fecal content. It has also been found that gastric mucosal is protected when the antiplatelet drug is enteric coating and it is avoided when proton-pump inhibitors (omeprazole) are associated to the antiplatelet drug.

Younossi et al. [16] found that superficial mucosal erosions may be responsible for severe bleeding when aspirin is associated to anticoagulant therapy. They also found that gastric bleeding usually takes place during the first 5 days after the beginning of the combined therapy. Altman et al. [17] after complete meta-analysis that thromboembolic prevention in patients with atrial fibrillation and mitral valve disease or prosthetic heart valves is equally effective when low level of anticoagulation (INR 2-3) plus $100 \mathrm{mg}$ /day of aspirin is used instead of higher doses. Furthermore, the risk of gastrointestinal bleeding is considerably prevented using lower doses.

More recent trials, mainly meta-analysis and guidelines [18]-[24], according to previous comments can be summarized in following conclusions:

1) Patients with atrial fibrillation alone (low flow blood rate) only need anticoagulant therapy with anti-vitamin $\mathrm{K}$ for an INR $=2-3$ [18] [20] [22]. Others prefer anticoagulation with, so called, new anticoagulants [20] [21] [22] in order to reduce the bleeding risk.

2) When atrial fibrillation is associated to other pathologies which cause turbulent high blood flow (mitral valve disease, prosthetic heart valves or complicated ischemic heart disease) one or two antiplatelet agents (aspirin, clopidogrel 
or both) [19] [20] [21] [22] [23] have to be associated the anticoagulant therapy. They also found that one antiplatelet is better that two because the antithrombotic effect is similar, and the bleeding risk is higher.

3) There is general agreement that double therapy significantly reduces the thrombo-embolic risk, but increases the incidence of major bleeding, mainly gastric.

4) When double or triple therapy is indicated the level of anticoagulation must be low (no higher than INR $=2-3$ ).

5) When antiplatelet agents are needed some investigators prefer clopidogrel to aspirin to reduce the bleeding risk [20] [21], but if the thromboembolic risk is exceedingly high others prefer aspirin [23].

6) The role of combined therapy in patients with atrial fibrillation at high risk of stroke (prior embolism, mitral stenosis or complicated ischemic heart disease) has not been unanimously advised in Guidelines: There is greater tendency to use double or triple therapy in USA than in Europe.

All investigators agree that aspirin is the main responsible for bleeding events, mainly upper gastrointestinal bleeding, and the risk is greatly reduced when the dose is lowered to $100 \mathrm{mg}$ /day. Furthermore, the use of enteric coating aspirin [20] considerably diminished the bleeding risk, according to previously cited gastroenterologists [12] [13] [14] [15] and the risk is totally avoided when omeprazole $20 \mathrm{mg}$ /day is associated.

Our NASPEAF randomized trial [25] [26] [27] analyzed the effect of antithrombotic therapy [25] in 958 patients with atrial fibrillation of whom 471 received combined therapy (anticoagulation (mean INR $=2.47$ ) and triflusal 600 $\mathrm{mg} /$ day) and 506 anticoagulant therapy alone (INR 2.3). Triflusal $600 \mathrm{mg} /$ day was used as antiplatelet. Its effect is similar to that of aspirin, but with lower prostacyclin inhibition, so greater doses are needed to obtain therapeutic level. The primary events were significantly lower in patients receiving combined therapy [25]. The incidence of major bleedings was also lower, although non-significant, in the combined group (17 versus 23), but 11 of 17 bleedings registered in this group were gastric and only 4 in the anticoagulant group. Gastric endoscopy performed in 10 of the 11 patients showed superficial mucosal gastric erosions, but ulcer was not detected in any of them.

Trying to avoid the high incidence of gastric bleeding in the combined therapy group we continued since 2001 to 2008 [28] control of 400 randomized patients of the NASPEAF trial distributed in two groups: group 1 composed by 165 of the anticoagulant arm (INR 2-3), and group 2 by 235 of the combined group (INR 1.9-2.5, triflusal $600 \mathrm{mg} /$ day and omeprazole $20 \mathrm{mg}$ ). 126 new cases entered in group 1, and 10 in group 2. Another two combined therapy groups were also followed: group 3 (120 cases), which received anticoagulation for INR 1.9-2.5, triflusal $300 \mathrm{mg} /$ day and omeprazole $20 \mathrm{mg}$ and group 4 (34 cases) treated with the same level of anticoagulation and enteric coating $100 \mathrm{mg}$ aspirin. The mean control time was 5.1 years. 
Only those patients controlled for a minimum period of 12 months were included for final analysis. Patients of groups 1 - 3 were controlled for a longer period (mean 49 months) and patients of group 4 for a mean period of 36.7 months. Systemic and final controls were realized during personal attendance in out-patients clinic, except a 5.9\%, in whom final control was made by telephone.

Eight major gastric bleedings were registered in combined therapy groups 2 and 3, all inpatients who were not yet receiving preventive treatment with omeprazole. Only one patient of group 2 presented one gastric bleeding after omeprazole therapy, but it was proved later the patient was not taken omeprazole when he suffered the bleeding. No gastric bleedings were registered in group 4, receiving entering coating aspirin 100.

After the publication of our NASPEAF trial, Rodney H. Falk [29] wrote: "Reconsidering Combined Antiplatelet and Anticoagulant Therapy in Atrial Fibrillation". Now that it has been proved that gastric bleeding is prevented by enteric coating antiplatelet tablets and avoiding with omeprazole, we can repeat "Reconsidering combined antiplatelet-anticoagulant therapy in Atrial Fibrillation complicated by Ischemic Heart Disease, Mitral Stenosis or Prosthetic Heart Valves".

\section{Conclusions}

1) Thrombus formation within the left atrium and the coronary or systemic arteries is the result of three main factors: a) formation of a fibrin net as the final step of the coagulation cascade with the contribution of thrombin, platelet activity and coagulation factors; b) platelet aggregation, secondary to platelet activity and prostaglandin contribution; c) the degree of blood flow rate: low flow, as occurs within the left atrium in patients with atrial fibrillation which facilitates coagulation and high turbulent flow, as occurs in patients with coronary or systemic stenosis, mitral stenosis or prosthetic heart valves which facilitates platelet aggregation.

2) The main therapy to prevent coagulation is based on the anticoagulant (antivitamin K) therapy, or more recently on the antithrombins therapy, which anticoagulant effect is not influenced by food absortion and there is no need for laboratory control. In combined therapy, usually antivitamin K-anticoagulants are used.

3) To prevent the consequences of high-turbulent flow we use antithromboxane-antiplatelet (mainly aspirin) or anti-prostacyclin (mainly clopidogrel).

4) Randomized trials showed a significant reduction of thromboembolic events when antivitamin $\mathrm{K}$-anticoagulants are combined with antiplatelets, but the risk of bleeding (mainly gastric) increased because of mucosal damage, which occurs mainly during the first 5 days of treatment. Gastroscopy usually shows gastric or duodenal ulcer in patients with major gastricbleeding, but our trials show that this event may happen with superficial gastric erosions when aspirin is combined with dose-depending anticoagulant therapy. 
5) Recent trails have progressively reduced the dose of anticoagulants to a mean INR level of 2.5 or less, and the dose of aspirin from initial 1000 to an actual 300 - $100 \mathrm{mg} /$ day. The risk of bleeding decreased with lower doses of both therapies, but a greater prevention was obtained when aspirin is enteric coating. Our experience is that gastric bleeding risk is totally avoided when the antiplatelet is given associated to omeprazole $20 \mathrm{mg}$ /day.

6) In our published experience combined therapy with an anticoagulant level for a mean INR of 2.17 and low dose of antithromboxan-antiplatelet therapy significantly reduced the thromboembolic risk and cardiac death. The risk of gastric bleeding was totally avoided associating omeprazole $20 \mathrm{mgs} /$ day or using enteric coating tablets when the dose of aspirin is $100 \mathrm{mg}$ day.

\section{Conflicts of Interest}

The authors declare no conflicts of interest regarding the publication of this paper.

\section{References}

[1] Macfarlane, R.G. (1964) An Enzyme Cascade in the Blood Clotting Mechanism and Its Function as a Biochemical Amplifier. Nature, 202, 498-499. https://doi.org/10.1038/202498a0

[2] Monroe, D.M. and Hoffman, M. (2006) What Does It Take to Make the Perfect Clot? Arteriosclerosis, Thrombosis, and Vascular Biology, 26, 41-48. https://doi.org/10.1161/01.ATV.0000193624.28251.83

[3] Schafer, A.I. (1994) Coagulation Cascade: An Overview. In: Loscalzo, J. and Schafer, A.I., Eds., Thrombosis and Haemorrhage, Blackwell Scientific, Boston, 3-12.

[4] Chesebro, J.H., et al. (1983) Trial of Combined Warfarin plus Dipyridamole or Aspirin Therapy in Prosthetic Heart Valve Replacement: Danger of Aspirin Compared with Dipyridamole. American Journal of Cardiology, 51, 1537-1541. https://doi.org/10.1016/0002-9149(83)90673-2

[5] Turpie, A.G., Gent, M., Laupacis, A., Latour, Y., Gunstensen, J., Basile, F., Klimek, M. and Hirsh, J. (1993) A Comparison of Aspirin with Placebo in Patients with Warfarin after Heart Valve Replacement. The New England Journal of Medicine, 329, 524-529. https://doi.org/10.1056/NEJM199308193290802

[6] Stroke Prevention in Atrial Fibrillation Investigators (1996) Adjusted-Dose Warfarin vs Lowintensity Fixed Dose Warfarin plus Aspirin for High Risk Patients with Atrial Fibrillation. Stroke Prevention in Atrial Fibrillation III, Randomized Clinical Trial. The Lancet, 348, 633-638.

[7] Gullov, A.L., Koefoed, B.G., Petersen, P., Pedersen, T.S., Andersen, E.D., Godtfredsen, J. and Boysen, G. (1998) Fixed Minidose Warfarin and Aspirin Alone and in Combination vs Adjusted Dose Warfarin for Stroke Prevention in Atrial Fibrillation. Second Copenhagen Atrial Fibrillation, Aspirin and Anticoagulation Study. Archives of Internal Medicine, 158, 1513-1521.

https://doi.org/10.1016/S0140-6736(96)03487-3

[8] Meschengieser, S.S., Fondevila, C.G., Frontroth, J., Santarelli, M.T. and Lazzari, M.A. (1997) Low-Intensity Oral Anticoagulation plus Low-Dose Aspirin versus High-Intensity Oral Anticoagulation Alone: A Randomized Trial in Patients with Mechanical Prosthetic Heart Valves. The Journal of Thoracic and Cardiovascular 
Surgery, 113, 910-916. https://doi.org/10.1001/archinte.158.14.1513

[9] Massel, D. and Little, S.H. (2001) Risk and Benefits of Adding Anti-Platelet Therapy to Warfarin Among Patients with Prosthetic Heart Valves: A Meta-Analysis. Journal of the American College of Cardiology, 37, 569-578.

https://doi.org/10.1016/S0022-5223(97)70264-2

[10] Lechat, P., Lardoux, H., Mallet, A., Sanchez, P., Derumeaux, G., Lecompte, T., Maillard, L., Mas, J.-L., Mentre, F., Pousset, F., Lacomblez, L., Pisica, G., Solbes-Latourette, S., Raynaud, P. and Chaumet-Riffaud, P. (2001) Anticoagulant (Fluindione) Aspirin Combination in Patiens with High-Risk Atrial Fibrillation. Cerebrovascular Diseases, 12, 245-252. https://doi.org/10.1016/S0735-1097(00)01135-9

[11] Dentali, F., Douketis, J.D., Lim, W. and Crowther, M. (2007) Combined Aspirin-Oral Anticoagulation Therapy Compared with Oral Anticoagulation Therapy Alone Among Patients at Risk for Cardiovascular Disease: A Meta-Analysis of Randomized Trials. Archives of Internal Medicine, 167, 117-124.

https://doi.org/10.1159/000047711

[12] Prichard, P.J., Kitchingman, G.K., Walt, R.P., Daneshmend, T.K. and Hawkey, C.J. (1989) Human Gastric Mucosal Bleeding Induced by Low Dose Aspirin, but Not Warfarin. BMJ, 298, 493-496. https://doi.org/10.1136/bmj.298.6672.493

[13] Hawthorne, A.B., Mahida, Y.R., Cole, A.T. and Hawkey, C.J. (1991) Aspirin-Induced Gastric Mucosal Damage: Prevention by Enteric-Coating and Relation to Prostaglandin Synthesis. British Journal of Clinical Pharmacology, 32, 77-83. https://doi.org/10.1111/j.1365-2125.1991.tb05616.x

[14] Bhatt, D.L., et al. (2010) Clopidogrel with or without Omeprazole in Coronary Artery Disease. The New England Journal of Medicine, 363, 1909-1917. https://doi.org/10.1056/NEJMoa1007964

[15] Chan, F.K., et al. (2005) Clopidogrel versus Aspirin and Esomeprazole to Prevent Recurrent Ulcer Bleeding. The New England Journal of Medicine, 352, 238-244. https://doi.org/10.1056/NEJMoa042087

[16] Younossi, Z.M., Strum, W.B., Schatz, R.A., Teirstein, P.S., Cloutier, D.A. and Spinks, T.J. (1997) Effect of Combined Anticoagulation and Low-Dose Aspirin Treatment on Upper Gastrointestinal Bleeding. Digestive Diseases and Sciences, 42, 79-82. https://doi.org/10.1023/A:1018833021039

[17] Altman, R., Rouvier, J. and Gurfinkel, E. (1995) Oral Anticoagulant Treatment with and without Aspirin. Thrombosis and Haemostasis, 74, 506-510. https://doi.org/10.1055/s-0038-1642729

[18] You, J.J., et al. (2012) Antithrombotic Therapy and Prevention of Thrombosis, 9th Ed: American College of Chest Physicians Evidence-Based Clinical Practice Guidelines. Chest, 141, 1129. https://doi.org/10.1378/chest.141.4.1129b

[19] Van Rein, N., Heide-Jørgensen, U., Lijfering, W.M., Dekkers, O.M., Sørensen, H.T. and Cannegieter, S.C. (2019) Major Bleeding Rates in Atrial Fibrillation Patients on Single, Dual and Tiple Antithrombotic Therapy. Results from a National Danish Cohort Study. Circulation, 139, 775-786. https://doi.org/10.1161/CIRCULATIONAHA.118.036248

[20] Haller, P.M., et al. (2019) Bleeding and Ischemic Outcomes in Patients Treated with Dual or Triple Antithrombotic Therapy: Systemic Review and Meta-Analysis. European Heart Journal-Cardiovascular Pharmacotherapy, 5, 226-236. https://doi.org/10.1093/ehjcvp/pvz021

[21] Hermans, H., et al. (2013) Antithrombotic Therapy in Patients with Heart Valve Prosthesis. Cardiology in Review, 21, 27-36. 
https://doi.org/10.1097/CRD.0b013e3182638578

[22] Lip, G.Y.H., et al. (2018) Antithrombotic Therapy for Atrial Fibrillation. Guidelines and Experts Panel Report. Chest, 154, 1121-1201.

https://doi.org/10.1016/j.chest.2018.07.040

[23] Whitlock, R.P., Sun, J.C., Fremes, S.E., Rubens, F.D. and Teoh, K.H. (2012) Antithrombotic and Thrombolytic Therapy for Valvular Disease: Antithrombotic Therapy and Prevention of Thrombosis, 9th Ed: American College of Chest Physicians Evidence-Based Clinical Practice Guidelines. Chest, 141, E576S-E600S. https://doi.org/10.1378/chest.11-2305

[24] Asencio, L.A., et al. (2014) Combining Antiplatelet and Antithrombotic Therapy (Triple Therapy): What Are the Risks and Benefits? The American Journal of Medicine, 127, 579-585. https://doi.org/10.1016/j.amjmed.2014.02.030

[25] Pérez-Gómez, F., et al. (2004) Comparative Effects of Antiplatelets, Study Anticoagulants or Combined Therapy in Patients with Valvular and Non-Valvular Atrial Fibrillation. A Randomized Multicenter Study. Journal of the American College of Cardiology, 44, 1557-1566. https://doi.org/10.1016/j.jacc.2004.05.084

[26] Pérez-Gómez, F., et al. (2006) Effect of Antithrombotic Therapy in Patients with Mitral Stenosis and Atrial Fibrillation: A Sub-Analysis of NASPEAF Randomized Trial. European Heart Journal, 27, 960-967. https://doi.org/10.1093/eurheartj/ehi667

[27] Pérez-Gomez, F., et al. (2007) Antithrombotic Therapy in Elderly Patients with Atrial Fibrillation: Effects and Bleeding Complications: A Stratified Analysis of the NASPEAF Randomized Trial. European Heart Journal, 28, 996-1003. https://doi.org/10.1093/eurheartj/ehl364

[28] Bover, R., et al. (2009) Long-Term Follow up of Atrial Fibrillation Patients in the NASPEAF Study. Prospective Follow-Up of Other Antiplatelet Treatments. Revista Española de Cardiología, 62, 992-1000. https://doi.org/10.1016/S1885-5857(09)73265-7

[29] Falk, R.H. (2004) Reconsidering Combined Antiplatelet and Anticoagulant Therapy in Atrial Fibrillation. Journal of the American College of Cardiology, 44, 1567-1569. https://doi.org/10.1016/j.jacc.2004.07.026 